\title{
Isolated Endoscopic Gluteus Medius Repair Can Achieve Successful Clinical Outcomes at Minimum 2-Year Follow-up
}

Mitchell B. Meghpara, M.D., Mitchell J. Yelton, B.S., Rachel M. Glein, B.S., Mohammad S. Malik, M.D., Philip J. Rosinsky, M.D., Jacob Shapira, M.D., David R. Maldonado, M.D., Hari K. Ankem, M.D., Ajay C. Lall, M.D., M.S., and Benjamin G. Domb, M.D.

\begin{abstract}
Purpose: To report on clinical presentations and outcomes in patients who underwent an isolated endoscopic gluteus medius (GM) repair. Methods: We retrospectively reviewed and prospectively collected data on patients who underwent a primary isolated endoscopic GM repair. Patients were included if the following patient-reported outcome scores were obtained preoperatively and at minimum 2-year follow-up: modified Harris Hip Score (mHHS), Non-arthritic Hip Score (NAHS), and visual analog scale (VAS) score. The minimal clinically important difference (MCID) for the mHHS, NAHS, and Hip Outcome Score-Sports Specific Scale was uniquely calculated for this study. Patients who underwent concomitant procedures including hip arthroplasty and hip arthroscopy were excluded. Results: A total of 26 hips met all inclusion and exclusion criteria, of which 23 hips (88.5\%) (22 patients) had a minimum 2-year follow-up. Nineteen hips had a partialthickness GM tear; 17 (89.5\%) were high-grade partial-thickness tears treated with a side-to-side repair through a transtendinous window. Four full-thickness tears with no retraction or fatty infiltration were able to be repaired endoscopically. The mHHS, NAHS, and VAS score all significantly improved $(P<.05)$ from baseline: The mHHS improved from 55.9 to 75.4 $(P=.005)$; the NAHS, from 58.8 to $79.2(P=.003)$; and the VAS score, from 5.9 to $3.6(P=.009)$. The MCID was calculated for the mHHS, NAHS, and Hip Outcome Score-Sports Specific Scale as 5.8, 7.1, and 11.0, respectively. Most patients achieved the MCID for all 3 patient-reported outcome scores. Conclusions: Appropriately selected patients without concomitant intra-articular hip pathology may achieve successful outcomes at a minimum 2-year follow-up after an isolated endoscopic GM repair. Most isolated endoscopic GM repairs were performed for partial-thickness GM tears. Level of Evidence: Level IV, case-series study.
\end{abstract}

G luteus medius (GM) tears typically present with lateral hip pain, abductor weakness, and gait abnormalities in women older than 50 years. ${ }^{1}$ When left untreated, GM tears can cause significant dysfunction and impairment of activities of daily living.

From the American Hip Institute Research Foundation, Chicago, Illinois, U.S.A. (M.B.M., M.J.Y., R.M.G., P.J.R., J.S., D.R.M., H.K.A., A.C.L., B.G.D.); AMITA Health St. Alexius Medical Center, Hoffman Estates, Illinois, U.S.A. (M.B.M., A.C.L., B.G.D.); University of Illinois at Chicago, Chicago, Illinois, U.S.A. (M.S.M.); and American Hip Institute, Chicago, Illinois, U.S.A. (A.C.L., B.G.D.).

The authors report the following potential conflicts of interest or sources of funding: M.B.M. receives educational support from Smith $\theta$ Nephew, Stryker, and Arthrex; receives food/beverage support from Smith $\theta$ Nephew, Stryker, and Arthrex; and travelllodging support from Smith $\theta$ Nephew, Stryker, and Arthrex, outside the submitted work. P.J.R. receives travel/lodging support from Arthrex, Stryker, and Smith $\theta$ Nephew and receives food/beverage support from Arthrex, Stryker, Smith $\theta$ Nephew, and Ossur, outside the submitted work. J.S. receives travel/lodging support from Arthrex, Stryker, and Smith $\theta$ Nephew and receives food/beverage support from Arthrex,
Voos et al. ${ }^{2}$ initially described endoscopic GM repair with successful outcomes in 10 patients after a shortterm mean follow-up period of 25 months. ${ }^{3}$ In this study, all 10 patients also underwent concomitant intraarticular treatment. Subsequent studies have shown

Stryker, Smith $\theta$ Nephew, and Ossur, outside the submitted work. D.R.M. receives travelllodging support from Arthrex, Stryker, and Smith $\theta$ Nephew, outside the submitted work; receives food/beverage support from Arthrex, Stryker, Smith $\theta$ Nephew, and Ossur, outside the submitted work; and is an editorial board of Arthroscopy. A.C.L. receives food/beverage support from Arthrex, Iroko, Smith $\theta$ Nephew, Stryker, Vericel, and Zimmer Biomet; receives grant support from Arthrex and Stryker; receives travel/lodging support from Arthrex and Stryker; receives consulting fees from Arthrex and Graymont Medical; and receives educational support from Medwest and Smith $\theta$ Nephew, outside the submitted work. A.C.L. is the Medical Director of Hip Preservation at St. Alexius Medical Center and the Clinical Instructor at the University of Illinois College of Medicine. B.G.D. reports that the American Orthopedic Foundation provides grant support that pays staff and expenses related to all research. B.G.D. receives royalties from Amplitude, Arthrex, DJO Global, Medacta, Stryker, and Orthomerica; receives research support from 


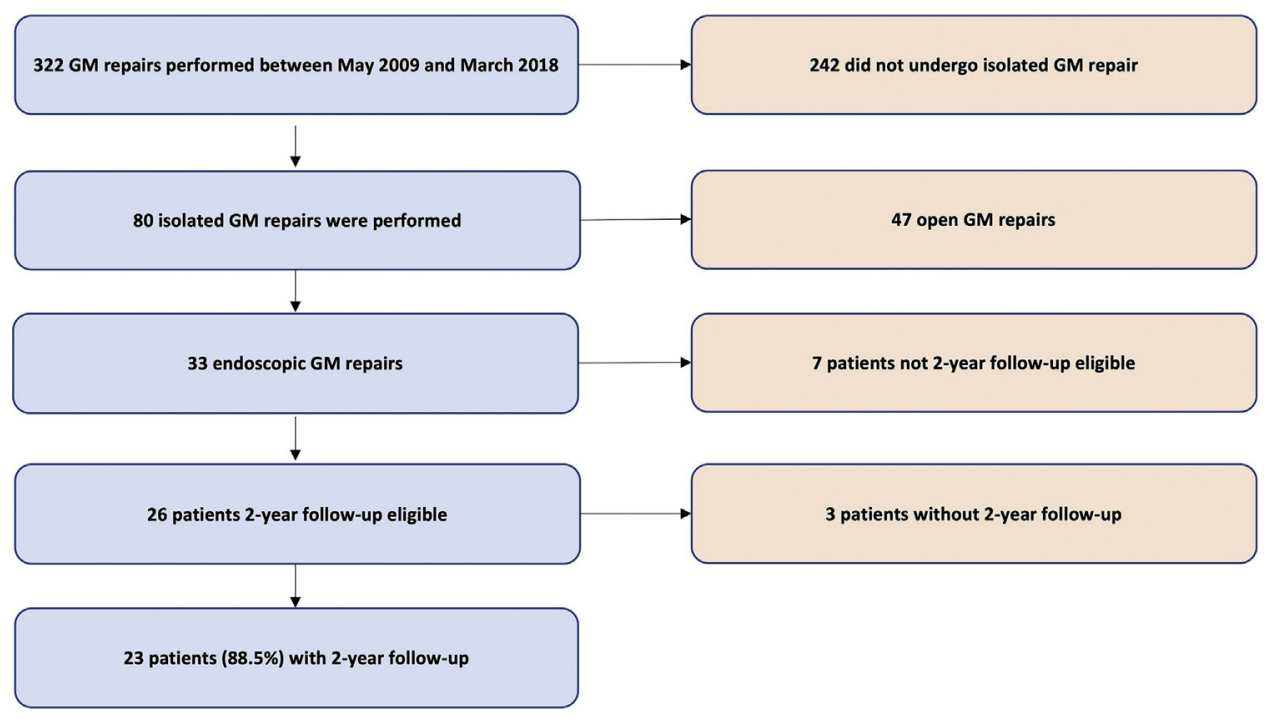

Figure 1. Flowchart showing patient selection process.

favorable improvements in multiple patient-reported outcomes (PROs) with larger cohorts at both short- and mid-term follow-up but also with concomitant intraarticular procedures performed. ${ }^{4,5}$ Recently, sustained survivorship and benefit were shown at minimum 5year follow-up after endoscopic GM repair along with at least a labral repair. ${ }^{6}$

The purpose of this study was to report on clinical presentations and outcomes in patients who underwent an isolated endoscopic GM repair. We hypothesized that patients would have favorable outcomes and minimal complications when treated in this fashion.

\section{Methods}

Data from a prospectively collected database from the study institution's hip preservation registry were retrospectively reviewed. All patients who underwent a primary endoscopic isolated GM repair performed by the senior author (B.G.D.) between May 2009 and March 2018 were considered eligible. Patients were included if the following PRO scores were obtained preoperatively and at minimum 2-year follow-up: modified Harris Hip Score (mHHS), ${ }^{7}$ Non-arthritic Hip Score (NAHS), ${ }^{8}$ and visual analog scale (VAS) score. ${ }^{9}$ Patients who underwent concomitant intra-articular procedures (total hip arthroplasty [THA] and hip arthroscopy) were excluded.

\section{Participation in American Hip Institute Hip Preservation Registry}

Although this study represents a unique analysis, data on some patients within this study may have been reported in other studies. All data collection received institutional review board approval.

\section{Preoperative Evaluation}

The senior author (B.G.D.) reviewed any pertinent medical history and performed a comprehensive physical examination of every patient before surgery. The findings of an assessment of gait, trochanteric tenderness, and abductor strength, as well as magnetic
Arthrex, Medacta, Stryker, and ATI Physical Therapy; receives educational support from Arthrex, Medacta, Stryker, Breg, Medwest Associates, St. Alexius Medical Center, and Ossur; receives consulting fees from Arthrex, Medacta, and Stryker; receives speaking fees from Arthrex; receives travel and lodging support from Arthrex, Medacta, and Stryker; receives food/beverage payments from Arthrex, DJO Global, Medacta, Stryker, and Zimmer Biomet; receives honoraria from Medacta; receives nonconsulting fees from Stryker; and has a medical directorship with St. Alexius Medical Center, outside the submitted work. B.G.D. has patents issued for the following: method and instrumentation for acetabular labrum reconstruction (8920497), licensed by Arthrex; adjustable multi-component hip orthosis (8708941), licensed by Orthomerica and DJO Global; and knotless suture anchors and methods of suture repair (9737292), licensed by Arthrex. B.G.D. is a board member of the American Hip Institute Research Foundation, Arthroscopy Association of North America Learning Center Committee, Journal of Hip Preservation Surgery, and
Arthroscopy; and has ownership interests in the American Hip Institute, Hinsdale Orthopedic Associates, Hinsdale Orthopedic Imaging, SCD\#3, North Shore Surgical Suites, and Munster Specialty Surgery Center. Full ICMJE author disclosure forms are available for this article online, as supplementary material.

Received June 2, 2021; accepted July 23, 2021.

Address correspondence to Benjamin G. Domb, M.D., American Hip Institute, 999 E Touhy Ave, Ste 450, Des Plaines, IL 60018, U.S.A. E-mail: DrDomb@americanhipinstitute.org

(C) 2021 THE AUTHORS. Published by Elsevier Inc. on behalf of the Arthroscopy Association of North America. This is an open access article under the CC BY-NC-ND license (http://creativecommons.org/licenses/by-nc-nd/4.0/).

2666-061X/21849

https://doi.org/10.1016/j.asmr.2021.07.026 
resonance imaging, were taken into consideration to confirm GM pathology. ${ }^{10,11}$ All patients underwent a wide-ranging course of nonoperative measures including but not limited to activity modification, antiinflammatory drugs, physical therapy, corticosteroid injections, and platelet-rich plasma injections. Patients were offered surgical intervention if these measures failed after at least 3 months. Those with partialthickness tears or full-thickness tears without significant retraction or fatty infiltration were indicated for an endoscopic repair.

\section{Surgical Technique}

Patients were positioned supine on a traction table with the operative leg in $20^{\circ}$ to $30^{\circ}$ of abduction. A distal anterolateral portal was established under fluoroscopic guidance and used as the viewing portal. Midanterior, anterolateral, and posterolateral accessory portals were established as working portals. After a trochanteric bursectomy was performed, the GM tendon was probed and evaluated for undersurface destabilization to confirm a tear. A suture staple technique was used to repair low-grade partial-thickness tears. ${ }^{12}$ High-grade partial-thickness tears were repaired in a side-to-side fashion through a transtendinous window. ${ }^{13}$ In instances in which a full-thickness tear was identified, a double-row suture bridge construct was used. ${ }^{14}$

\section{Rehabilitation}

Patients who underwent an endoscopic suture staple repair were instructed to wear a fitted X-Act ROM hip brace (DJO Global, Vista, CA) and use crutches with partial weight bearing $(20 \mathrm{lb})$ for 2 weeks and to begin physical therapy immediately after surgery. Patients who underwent an endoscopic side-to-side repair or endoscopic double-row suture bridge repair were instructed to wear the brace and use crutches for 6 weeks and to begin physical therapy at 6 weeks postoperatively.

\section{Surgical Outcomes}

Patients were prospectively assessed with questionnaires preoperatively and at 3 months after surgery, then annually. Surveys were collected at office visits,

Table 1. Demographic Characteristics

\begin{tabular}{lc}
\hline \multicolumn{1}{c}{ Characteristic } & Data \\
\hline Hips included in study, $\mathrm{n}$ & 23 \\
Left & 11 \\
Right & 12 \\
Sex, $\mathrm{n}(\%)$ & $22(95.7)$ \\
$\quad$ Female & $1(4.3)$ \\
$\quad$ Male & $62.7 \pm 10.1(36.9-74.4)$ \\
Age at surgery, mean \pm SD (range), yr & $29.9 \pm 4.2(21.4-37.7)$ \\
BMI, mean \pm SD (range) & $38.3 \pm 19.2(24.0-84.5)$ \\
Follow-up time, mean \pm SD (range), mo &
\end{tabular}

Table 2. Intraoperative Findings

\begin{tabular}{lr}
\hline GM Tear & $\mathrm{n}(\%)$ \\
\hline Partial tear & $19(82.6)$ \\
Low grade $(<50 \%)$ & $2(10.5)$ \\
High grade $(>50 \%)$ & $17(89.5)$ \\
Full tear & $4(17.4)$ \\
\hline GM, gluteus medius.
\end{tabular}

GM, gluteus medius.

through telephone interviews, or via encrypted e-mails. All preoperative questionnaires included the following PRO scores: mHHS, NAHS, Hip Outcome Score-Sports Specific Scale (HOS-SSS), ${ }^{15}$ and VAS score. Additionally, postoperative questionnaires included the International Hip Outcome Tool 12 (iHOT-12), ${ }^{16}$ the mental and physical components of the 12-Item Short Form Health Survey (SF-12M and SF-12P, respectively), and the mental and physical components of the Veterans RAND 12-Item Health Survey (VR-12M and VR-12P, respectively). ${ }^{17}$ Patient satisfaction was recorded on a scale of 0 to 10 , with 10 indicating extreme satisfaction. The minimal clinically important difference (MCID) was calculated specifically for this study population as described by Norman et al. ${ }^{18}$ for the following PRO scores: mHHS, NAHS, and HOS-SSS. A subanalysis comparing partial- versus full-thickness tears was performed. Furthermore, complications and rates of revision arthroscopy and conversion to THA were reported.

\section{Statistical Analysis}

The Real Statistics add-in package in Microsoft Excel (Microsoft, Redmond, WA) was used for all statistical analyses. Normality and variance were assessed using the Shapiro-Wilk test and $F$ test, respectively. The Fisher exact test or $\chi^{2}$ test was used for all categorical data, whereas continuous data were analyzed using the 2 -tailed $t$ test or, when appropriate, its nonparametric equivalent. The level of statistical significance was defined as $P<.05$.

\section{Results}

\section{Patient Characteristics}

A total of 26 hips met all inclusion and exclusion criteria, of which 23 hips $(88.5 \%)$ (22 patients) had a minimum 2 -year follow-up. The patient selection process is outlined in Fig 1. Female patients comprised $95.7 \%$ of the study cohort, and the average follow-up period was 38.3 months (range, 24.0-84.5 months).

Table 3. Gluteus Medius Procedures Performed

\begin{tabular}{lc}
\hline \multicolumn{1}{c}{ Repair Technique } & $\mathrm{n}(\%)$ \\
\hline Transtendinous technique & $18(78.3)$ \\
Suture staple & $1(4.3)$ \\
Double-row suture bridge & $4(17.4)$ \\
\hline
\end{tabular}




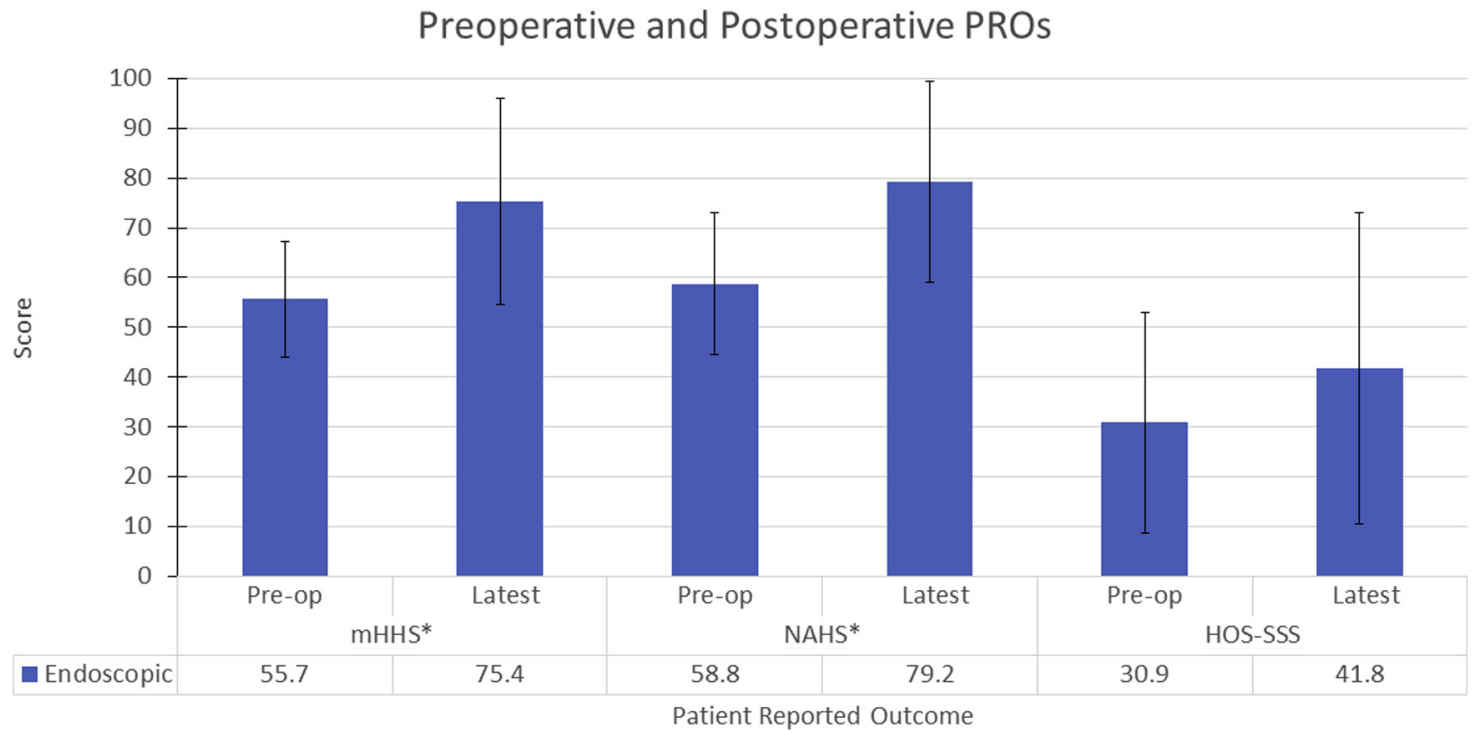

Fig 2. Patient-reported outcome (PRO) scores from preoperatively (Pre-op) to latest follow-up at minimum 2 of years. Asterisks indicate $P<.05$. (HOS-SSS, Hip Outcome Score-Sports Specific Scale; mHHS, modified Harris Hip Score; NAHS, Non-arthritic Hip Score.)

Complete patient demographic characteristics are outlined in Table 1.

\section{Physical Examination Findings}

Preoperatively, all 23 patients reported trochanteric tenderness whereas $10(43.5 \%)$ were found to have a positive Trendelenburg sign. A positive Trendelenburg sign was indicated when a patient's contralateral hip sagged or when the trunk swayed to the contralateral side on a single-leg stance. Only 1 tear $(4.3 \%)$ was the result of an associated injury. Patients reported symptoms lasting an average of $45.7 \pm 39.0$ months (range, 5.0-168.0 months).

\section{Intraoperative Findings and Procedures}

Nineteen hips had a partial-thickness GM tear; 17 $(89.5 \%)$ were high-grade partial-thickness tears treated with a side-to-side repair through a transtendinous window. An additional side-to-side repair was performed for a low-grade partial-thickness tear owing to poor tissue quality, which needed further undersurface debridement through a transtendinous window. Four

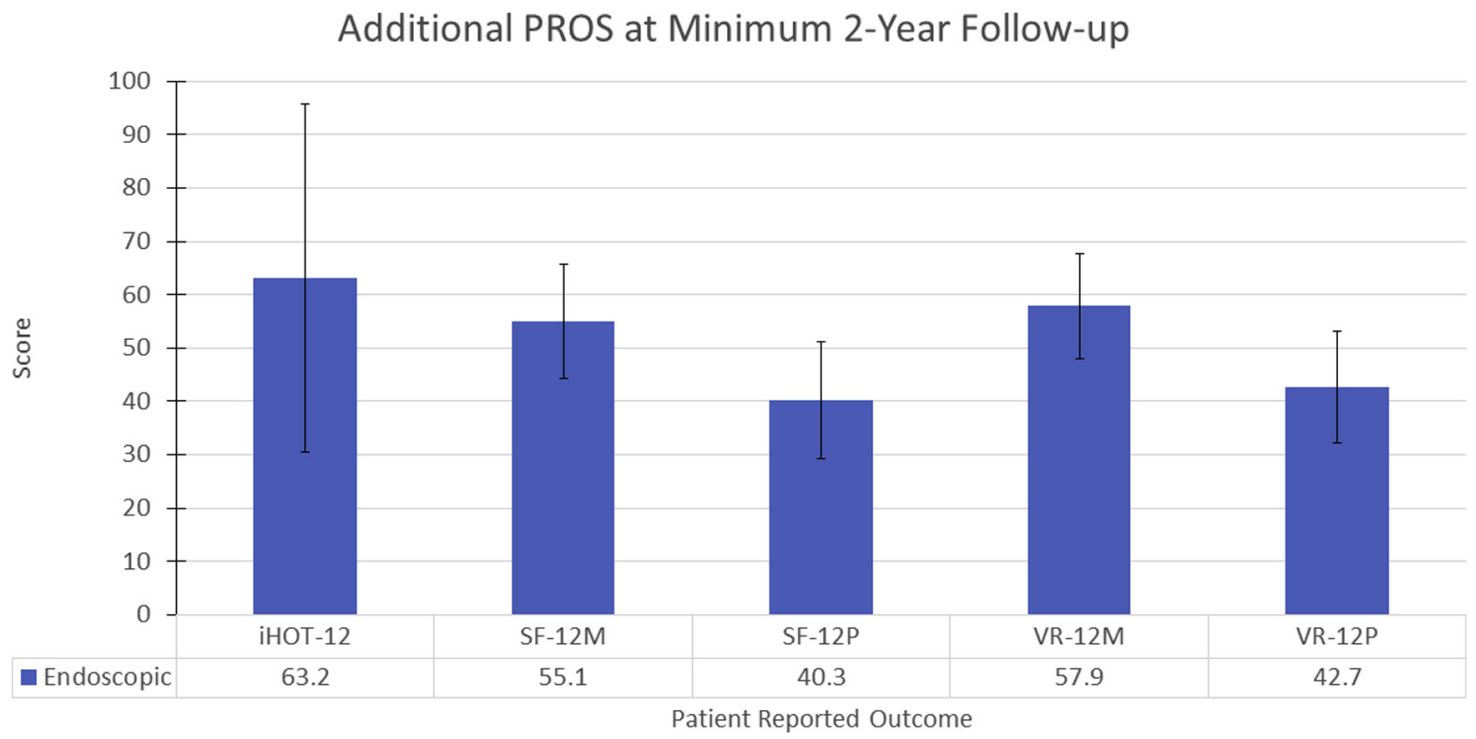

Fig 3. Additional patient-reported outcome scores (PROS) collected at minimum 2-year follow-up. (iHOT-12, International Hip Outcome Tool-12; SF-12M, 12-Item Short Form Health Survey mental component; SF-12P, 12-Item Short Form Health Survey physical component; VR-12M, Veterans RAND 12-Item Health Survey mental component; VR-12P, Veterans RAND 12-Item Health Survey physical component.) 


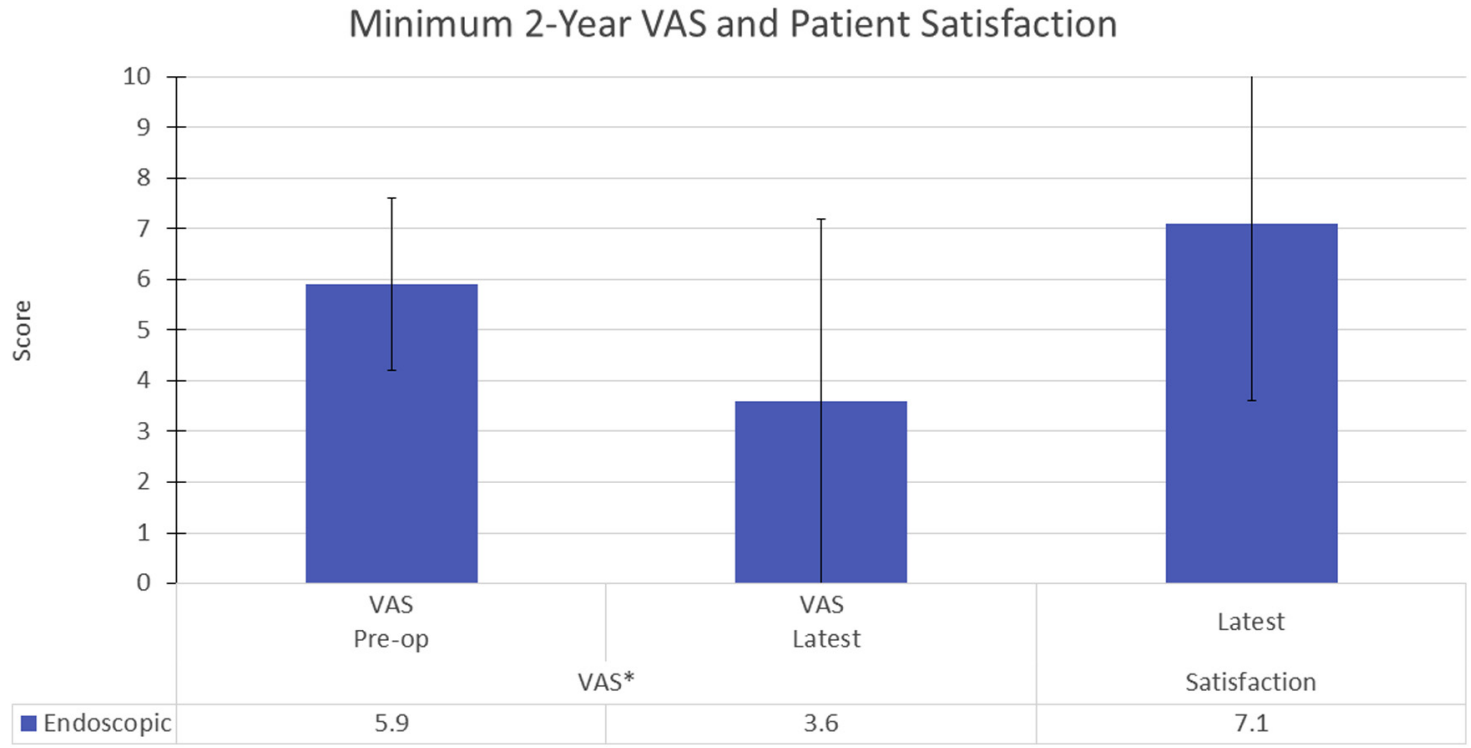

Fig 4. Visual analog scale (VAS) scores and patient satisfaction at minimum 2 -year follow-up. Asterisks indicate $P<.05$. (Pre-op, preoperative.)

full-thickness tears with no retraction or fatty infiltration were able to be repaired endoscopically (Tables 2 and 3).

\section{Clinical Outcomes}

The mHHS, NAHS, and VAS score all significantly improved $(P<.05)$ from baseline: The mHHS improved from 55.9 to $75.4(P=.005)$; the NAHS, from 58.8 to 79.2 $(P=.003)$; and the VAS score, from 5.9 to $3.6(P=.009)$. Similarly, patients reported improvements in the HOSSSS, albeit not significant (from 30.9 to $41.8, P=.275$ ). Fig 2 illustrates the changes in the PRO scores from baseline. All PRO scores at latest follow-up are summarized in Fig 3. The patient satisfaction score was reported as 7.1 at latest follow-up (Fig 4). The MCID was calculated for the MHHS, NAHS, and HOS-SSS as 5.8, 7.1, and 11.0, respectively. Most patients achieved the MCID for all 3 PRO scores (Table 4).

\section{Complications and Secondary Surgical Procedures}

One patient reported severe anterior spasms and lateral thigh numbness. Additionally, 3 hips treated with an endoscopic side-to-side repair required revision surgery at an average of 17.4 months postoperatively because of a retear. Unrelated to the GM repair, 1 hip underwent a subsequent secondary operation owing to

Table 4. MCID in Outcome Scores of Patients

\begin{tabular}{lr}
\hline \multicolumn{1}{c}{ Score } & $\mathrm{n}(\%)$ \\
\hline mHHS (MCID $\geq 5.8)$ & $12(57.1)$ \\
NAHS (MCID $\geq 7.1)$ & $14(73.7)$ \\
HOS-SSS (MCID $\geq 11.0)$ & $5(50.0)$ \\
\hline HOS-SSS, Hip Outcome Score-Sports Specific Scale; MCID, mini- \\
mal clinically important difference; mHHS, modified Harris Hip Score; \\
NAHS, Non-arthritic Hip Score.
\end{tabular}

continued hip pain from symptomatic femoroacetabular impingement with a labral tear at 13.3 months. Furthermore, 2 patients underwent conversion to THA at 9.2 and 12.0 months because of the development of osteoarthritis postoperatively.

\section{Tear Type Subanalysis}

Patients who underwent an endoscopic repair for a partial-thickness tear experienced significant improvements in the NAHS and VAS score $(P=.041$ and $P=.013$, respectively). The mHHS and HOS-SSS also improved in this subcohort, although not significantly $(P=.051$ and $P=.844$, respectively). Patients with full-thickness tears showed statistically significant improvements in the mHHS and NAHS ( $P=.023$ and $P=.007$, respectively). The VAS score and HOS-SSS also improved, although not significantly $(P=.625$ and $P=.096$, respectively). Except for the SF-12M score, all PRO scores were comparable between the partial-thickness and full-thickness cohorts $(P>.05)$. For the SF-12M, the full-thickness cohort showed significantly higher outcome scores (62.0 vs $53.2, P=.012)$. The results are summarized in Figures 5, 6, and 7 .

\section{Discussion}

This study shows that isolated endoscopic GM repair results in high patient satisfaction and improvement in multiple validated PRO scores at minimum 2-year follow-up. Furthermore, patients with partialthickness tears compared similarly with those with full-thickness tears at latest follow-up.

Overall, endoscopic GM repair has been proved to provide excellent benefit. Many prior studies have reported successful endoscopic repair with concomitant 


\section{Partial vs. Full-Thickness Sub-analysis of Preoperative and Postoperative PROs}

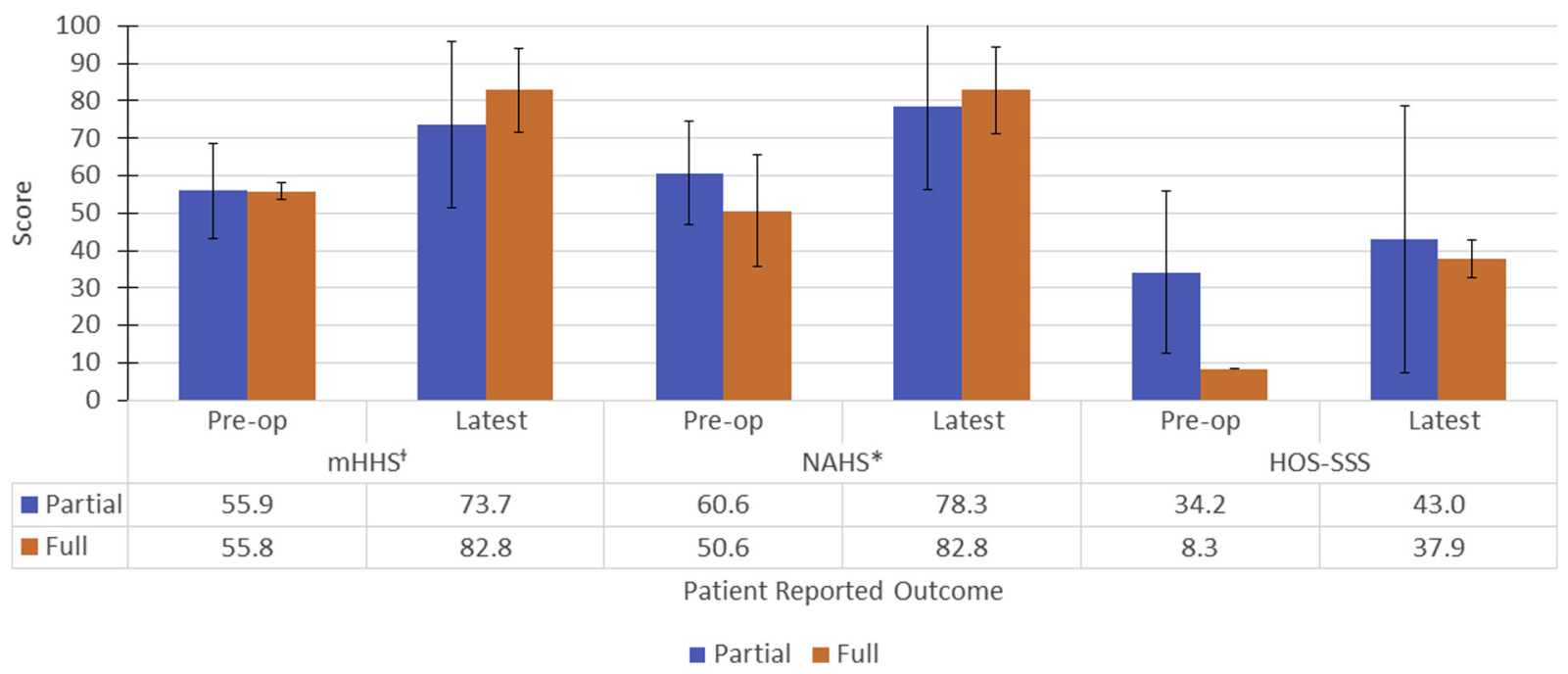

Fig 5. Partial- versus full-thickness tear subanalysis of patient-reported outcome (PRO) scores from preoperatively (Pre-op) to latest follow-up at minimum of 2 years. An asterisk indicates $P<.05$ for both subcohorts; a dagger indicates $P<.05$ for the fullthickness cohort. (HOS-SSS, Hip Outcome Score-Sports Specific Scale; mHHS, modified Harris Hip Score; NAHS, Non-arthritic Hip Score.)

arthroscopy to address coexisting femoroacetabular impingement pathology. Perets et al., ${ }^{4}$ Hartigan et al., ${ }^{19}$ and Domb et al. ${ }^{20}$ all reported significant improvements within their cohorts for the HOS-SSS, with mean scores of 66.4, 67.3, and 79.7, respectively, at latest follow-up. We found patients undergoing isolated endoscopic repair to have a mean HOS-SSS of 41.8 without significant improvement at latest follow-up. Additionally, the 3 aforementioned studies showed mean VAS scores at latest follow-up of 2.6, 2.7, and 1.4, respectively, versus 3.6 in our cohort. Finally, the mean satisfaction scores at latest follow-up were 8.4, 7.5, and 9.1 , respectively, versus 7.1 in this study. Evaluating the hip intra-articularly proves to be useful from both a

\section{Partial vs. Full-Thickness Tear Sub-Analysis of Additional PROs at Minimum 2-Year Follow-Up}

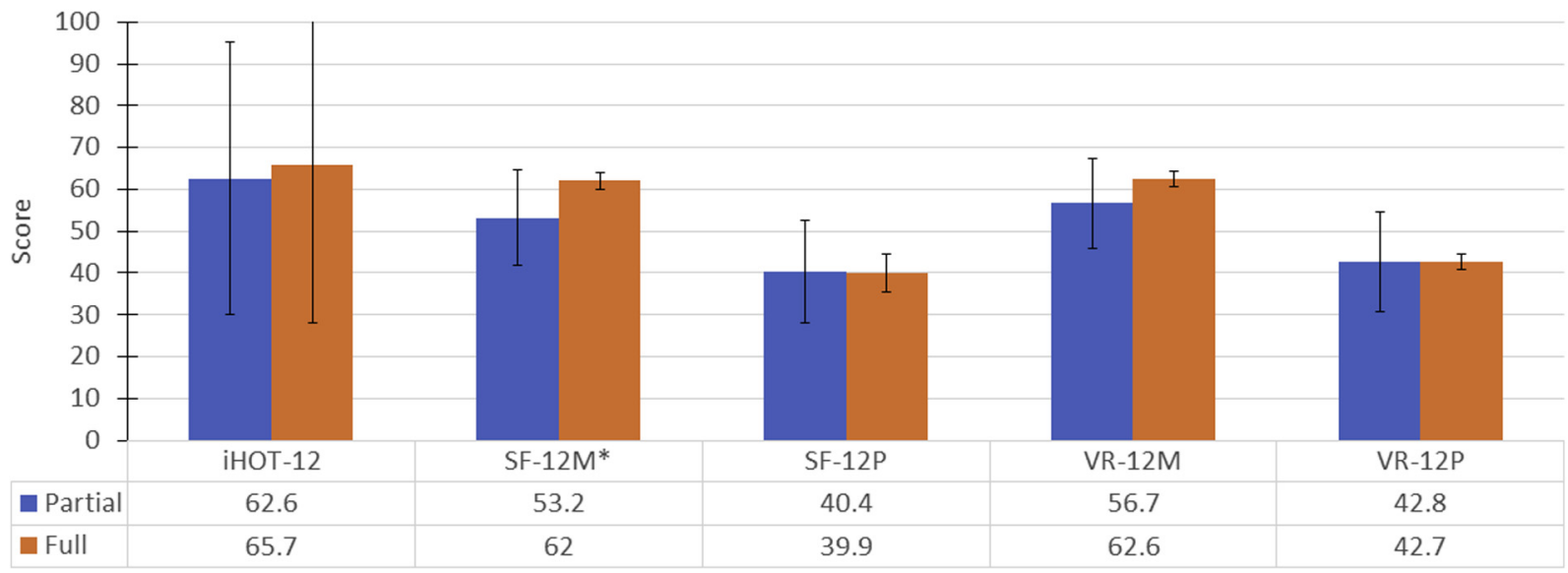

Patient Reported Outcome

- Partial a Full

Fig 6. Partial- versus full-thickness tear subanalysis of additional patient-reported outcome (PRO) scores at minimum 2 -year follow-up. An asterisk indicates $P<.05$. (iHOT-12, International Hip Outcome Tool-12; SF-12M, 12-Item Short Form Health Survey mental component; SF-12P, 12-Item Short Form Health Survey physical component; VR-12M, Veterans RAND 12-Item Health Survey mental component; VR-12P, Veterans RAND 12-Item Health Survey physical component.) 


\section{Partial vs. Full-Thickness Sub-Analysis of VAS and Patient Satisfaction} at Minimum 2-Year Follow-Up

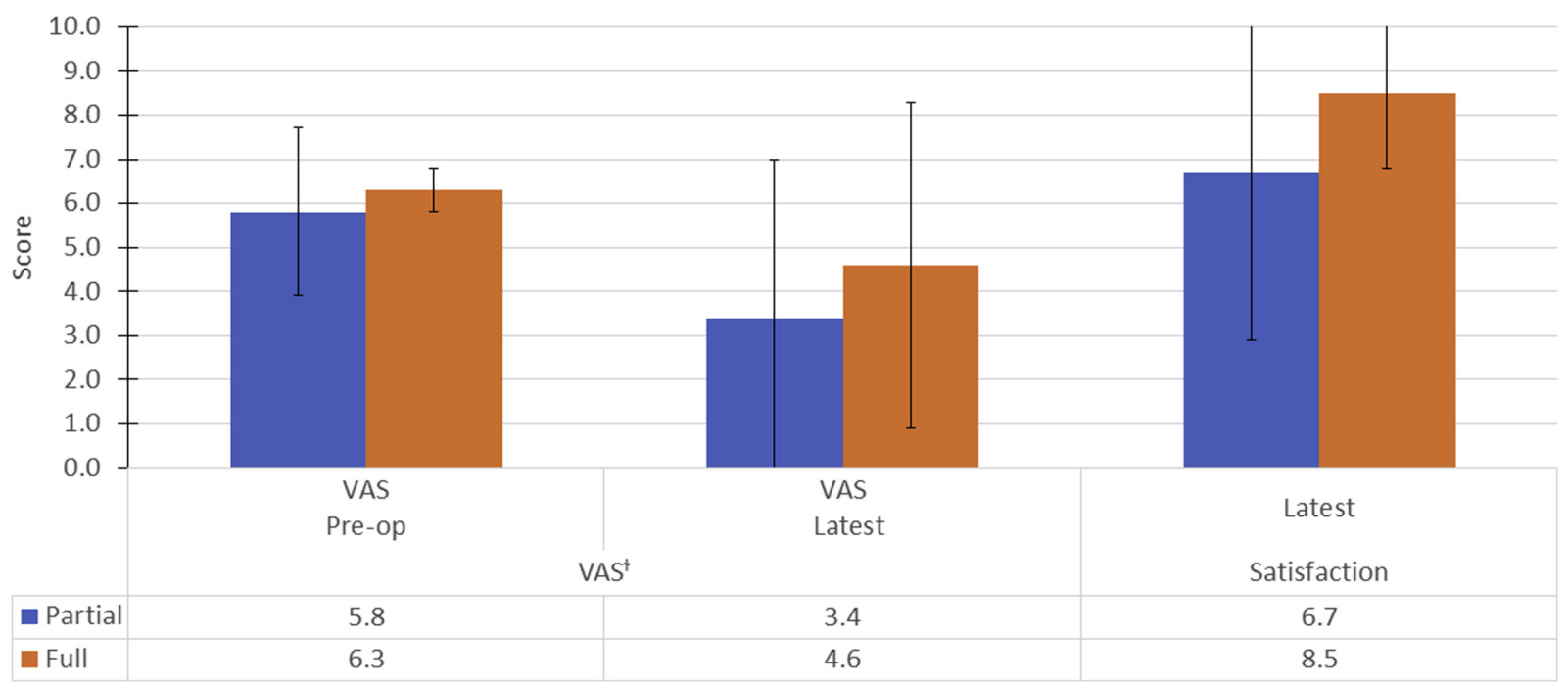

Fig 7. Partial- versus full-thickness tear subanalysis of visual analog scale (VAS) scores and patient satisfaction at minimum 2year follow-up. An dagger indicates $P<.05$ for the partial-thickness cohort. (Pre-op, preoperative.)

diagnostic and therapeutic standpoint to assess and treat any pathology accordingly. Thus, it may be plausible to surmise that superior improvements in the HOS-SSS, VAS score, and satisfaction score were achieved in prior studies by addressing associated intraarticular pathology.

An interesting finding in our study was that 3 patients with high-grade partial-thickness tears treated endoscopically with a side-to-side repair experienced continued pain postoperatively, which required reoperation for a retear. Two of these patients were women, aged 69 and 71 years at the time of surgery. GM repairs pose a unique challenge in which GM tears commonly develop in older women over the age of 50 years. ${ }^{1}$ Inert biology and osteopenia may contribute to reduced healing potential and diminished suture anchor pullout strength. $^{21}$

Calculating the MCID helps to identify the significance of PRO scores from a clinical standpoint. The MCID is dependent on patient characteristics such as socioeconomic status, disease severity, and patient expectations. ${ }^{22}$ Thus, in this study, we calculated a unique MCID specific to our patient population according to the distribution-based half-standard deviation method described by Norman et al. ${ }^{18}$ A significant proportion of our cohort achieved the MCID for the mHHS, NAHS, and HOS-SSS, thereby showing impactful clinical improvement.

This study's strengths include it being among the first studies to report on clinical outcomes in patients undergoing an isolated endoscopic GM repair performed by a single surgeon. In addition, patient satisfaction scores,
VAS scores for pain, and multiple validated hip-specific PRO scores were all used to evaluate outcomes. Moreover, no concomitant procedures were performed, which helps eliminate possible confounding effects on the surgical procedure's overall result. Finally, meaningful clinical improvement was determined by calculation of the MCID for the mHHS, NAHS, and HOS-SSS, exclusive to this study's patient population.

\section{Limitations}

There are noteworthy limitations in this study to address. First, selection bias can occur in retrospective studies; however, all data were collected prospectively to help minimize this bias. Second, the generalizability of our results may be limited because all operations in this study were performed at the study institution by a single surgeon specializing in hip preservation. Third, owing to small patient sample sizes, our subanalysis comparing tear type may not have been adequately powered to detect statistically significant differences. Finally, conversion to THA was an endpoint outcome; thus, postoperative scores for patients who underwent conversion to THA were not included in the final analysis.

\section{Conclusions}

Appropriately selected patients without concomitant intra-articular hip pathology may achieve successful outcomes at a minimum 2-year follow-up after an isolated endoscopic GM repair. Most isolated endoscopic GM repairs were performed for partial-thickness GM tears. 


\section{References}

1. Lindner D, Shohat N, Botser I, Agar G, Domb BG. Clinical presentation and imaging results of patients with symptomatic gluteus medius tears. J Hip Preserv Surg 2015;2: 310-315.

2. Voos JE, Rudzki JR, Shindle MK, Martin H, Kelly BT. Arthroscopic anatomy and surgical techniques for peritrochanteric space disorders in the hip. Arthroscopy 2007;23:1246.el-1246.e5.

3. Voos JE, Shindle MK, Pruett A, Asnis PD, Kelly BT. Endoscopic repair of gluteus medius tendon tears of the hip. Am J Sports Med 2009;37:743-747.

4. Perets I, Mansor Y, Yuen LC, Chen AW, Chaharbakhshi EO, Domb BG. Endoscopic gluteus medius repair with concomitant arthroscopy for labral tears: A case series with minimum 5-year outcomes. Arthroscopy 2017;33:2159-2167.

5. Chandrasekaran S, Gui C, Hutchinson MR, Lodhia P, Suarez-Ahedo C, Domb BG. Outcomes of endoscopic gluteus medius repair: Study of thirty-four patients with minimum two-year follow-up. J Bone Joint Surg Am 2015;97:1340-1347.

6. Meghpara MB, Yelton MJ, Annin S, et al. Mid-term outcomes of endoscopic gluteus medius repair with concomitant arthroscopic labral treatment: A propensity-matched controlled study. Arthroscopy 2020;36:2856-2865.

7. Aprato A, Jayasekera N, Villar RN. Does the modified Harris Hip Score reflect patient satisfaction after hip arthroscopy? Am J Sports Med 2012;40:2557-2560.

8. Christensen CP, Althausen PL, Mittleman MA, Lee J, McCarthy JC. The Nonarthritic Hip Score: Reliable and validated. Clin Orthop 2003;(406):75-83.

9. Chandrasekaran S, Gui C, Walsh JP, Lodhia P, SuarezAhedo C, Domb BG. Correlation between changes in visual analog scale and patient-reported outcome scores and patient satisfaction after hip arthroscopic surgery. Orthop J Sports Med 2017;5:2325967117724772.

10. Hartigan DE, Perets I, Walsh JP, Domb BG. Imaging of abductor tears: Stepwise technique for accurate diagnosis. Arthrosc Tech 2017;6:e1523-el527.

11. Lachiewicz PF. Abductor tendon tears of the hip: Evaluation and management. J Am Acad Orthop Surg 201 1;19:385-391.
12. Hartigan DE, Mansor Y, Perets I, Walsh JP, Mohr MR, Domb BG. Knotless "suture staple" technique for endoscopic partial thickness abductor tendon repair. Arthrosc Tech 2018;7:e975-e980.

13. Domb BG, Nasser RM, Botser IB. Partial-thickness tears of the gluteus medius: Rationale and technique for transtendinous endoscopic repair. Arthroscopy 2010;26: 1697-1705.

14. Domb BG, Carreira DS. Endoscopic repair of full-thickness gluteus medius tears. Arthrosc Tech 2013;2:e77-e81.

15. Martin RL, Philippon MJ. Evidence of validity for the Hip Outcome Score in hip arthroscopy. Arthroscopy 2007;23: 822-826.

16. Griffin DR, Parsons N, Mohtadi NGH, Safran MR. Multicenter Arthroscopy of the Hip Outcomes Research Network. A short version of the International Hip Outcome Tool (iHOT-12) for use in routine clinical practice. Arthroscopy 2012;28:611-616. quiz 616-618.

17. Kwon JY, Sawatzky R. Examining gender-related differential item functioning of the Veterans Rand 12-item Health Survey. Qual Life Res 2017;26: 2877-2883.

18. Norman GR, Sloan JA, Wyrwich KW. Interpretation of changes in health-related quality of life: The remarkable universality of half a standard deviation. Med Care 2003;41:582-592.

19. Hartigan DE, Perets I, Ho SW, Walsh JP, Yuen LC, Domb BG. Endoscopic repair of partial-thickness undersurface tears of the abductor tendon: Clinical outcomes with minimum 2-year follow-up. Arthroscopy 2018;34: 1193-1199.

20. Domb BG, Botser I, Giordano BD. Outcomes of endoscopic gluteus medius repair with minimum 2-year follow-up. Am J Sports Med 2013;41:988-997.

21. Putnam JG, Chhabra A, Castañeda P, et al. Does greater trochanter decortication affect suture anchor pullout strength in abductor tendon repairs? A biomechanical study. Am J Sports Med 2018;46: 1668-1673.

22. Sedaghat AR. Understanding the minimal clinically important difference (MCID) of patient-reported outcome measures. Otolaryngol Neck Surg 2019;161:551-560. 